11 Frippiat F, Bergiers C, Michel C, et al. Severe bilateral optic neuritis associated with prolonged linezolid therapy. $J$ Antimicrob Chemother 2004; 53: 1114-1115.

12 Seung KJ, Omatayo DB, Keshavjee S, et al. Early outcomes of MDR-TB treatment in a high HIV-prevalence setting in Southern Africa. PLoS One 2009; 4: e7186.

13 de Jager P, van Altena R. Hearing loss and nephrotoxicity in long-term aminoglycoside treatment in patients with tuberculosis. Int J Tuberc Lung Dis 2002; 6: 622-627.

14 Srivastava S, Peloquin CA, Sotgiu G, et al. Therapeutic drug management: is it the future of multidrug-resistant tuberculosis treatment? Eur Respir J 2013; 42: 1449-1453.

15 Bolhuis MS, van Altena R, van Soolingen D, et al. Clarithromycin increases linezolid exposure in multidrug-resistant tuberculosis patients. Eur Respir J 2013; 42: 1614-1621.

\title{
Is Europe ready to reach tuberculosis elimination? An outbreak report from Southern Italy
}

To the Editor:

In July 2014, in Rome, the European Respiratory Society and the World Health Organization (WHO) developed a global framework for tuberculosis (TB) elimination in low TB incidence countries, focussed on the concept of TB elimination (fewer than one TB case per million population) [1-5]. Recent studies demonstrated that several "areas" relevant for TB elimination are not fully covered in Europe and in Italy [6, 7].

The aim of the present study was to describe a recent TB outbreak in Puglia (Southern Italy), discuss the practical problems faced by clinicians in managing it correctly, and identify the critical organisational areas impeding proper implementation of WHO and ECDC (European Centre for Disease Prevention and Control) recommendations $[8,9]$.

When deviations from established recommendations $[8,9]$ were observed, a symbol $\left({ }^{d}\right)$ is added in the text.

On December 27, 2013, a 4-year-old child (case A) (fig. 1) was admitted because of long-lasting fever (since November 20) after mononucleosis was excluded. On December 28, chest radiography showed interstitial pneumonia (for which ineffective antibiotic treatment, ceftriaxone plus amikacin, was prescribed) while being tuberculin skin test (TST) negative. Culture was requested (later being positive) and a blood specimen for interferon- $\gamma$ release assay (IGRA) (Quantiferon TB Gold; Cellestis, Carnegie, Australia) was sent to a different laboratory (the only one within the province where the test is available), which resulted positive on January 3, 2014. The computed tomography (CT) confirmed a miliary picture. Standard treatment was initiated with four first-line drugs (isoniazid $(\mathrm{H})$, rifampicin $(\mathrm{R})$, pyrazinamide $(\mathrm{Z})$ and ethambutol (E)). The lack of access to rapid diagnostics $\left({ }^{\mathrm{d} 1}\right)$ in a sputum smear negative case, necessitating a wait for solid culture results $\left({ }^{\mathrm{d} 2}\right)$, contributed to delayed diagnosis, although after admission, adequate treatment was rapidly prescribed. The antibiotic treatment, although including a drug active against Mycobacterium tuberculosis, was considered essential to treat pneumonia.

All adult contacts underwent TST (January 6-10, 2014) and all children attending the same nursery school had IGRA testing (from January 13). Among family members and close contacts of the first circle, 28 adults were tested and three resulted as TST positive and chest radiography negative. Out of 24 health staff tested, all were TST negative. Two children (contacts of case A during his admission) were TST and IGRA negative.

Case A's father recalled that in September 2013, the entire family attended a parish party (involving the members of a local immigrants' association) and a 27 -years-old man had a severe cough. The local health department recorded that in October, a pulmonological medical examination was performed because of bronchitis reported by a man born in Pakistan. He was traced (case B), and underwent chest radiography (multiple lesions on the right lung) and bacteriology (positive for alcohol- and acid-fast bacilli (AFB)). Later, culture resulted positive for M. tuberculosis, while his 24-locus mycobacterial interspersed repetitive units (MIRU) variable number tandem repeats (VNTR) (performed at S. Raffaele Institute, Milan, Italy, in November 2014) resulted different from that of Case A. 


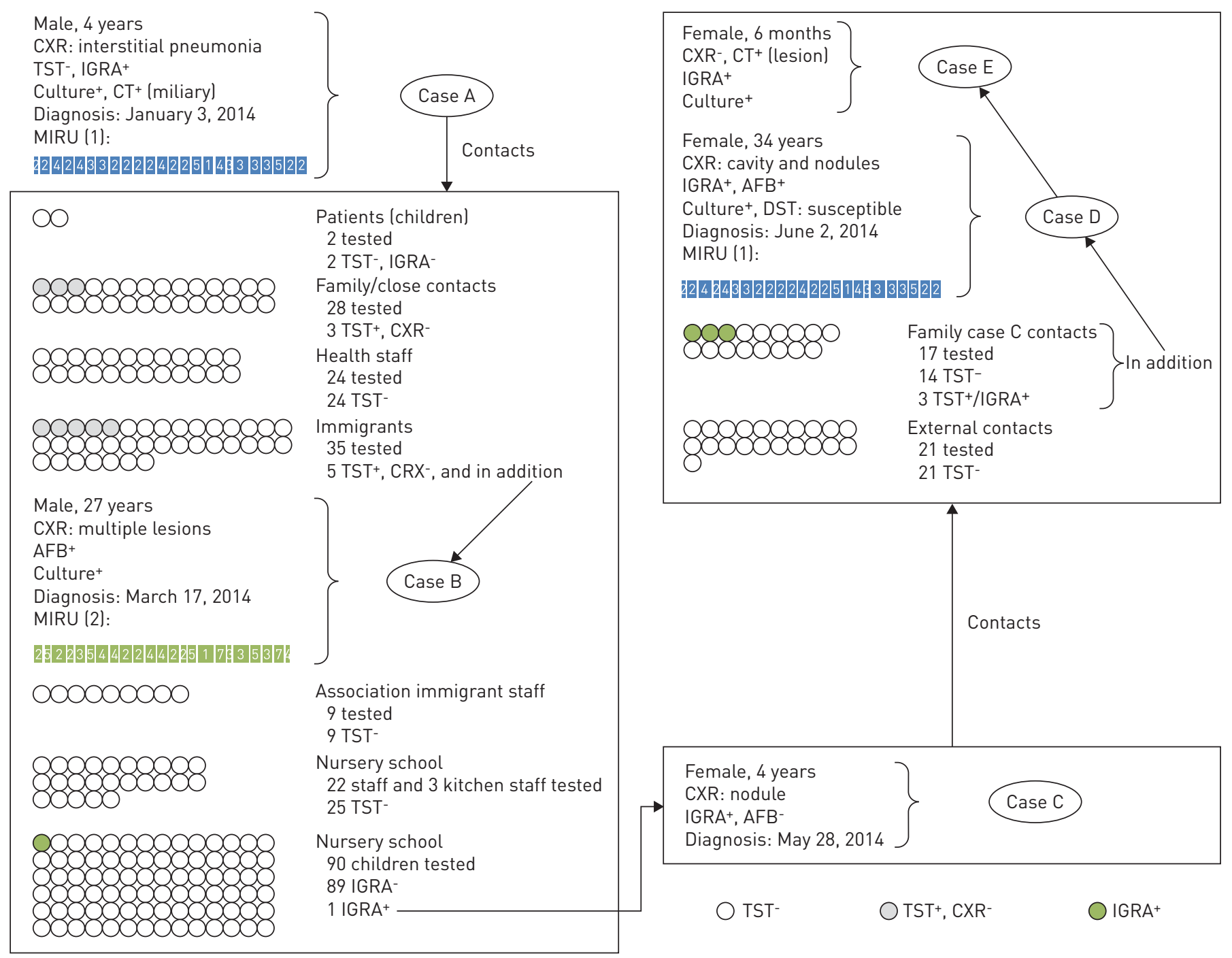

FIGURE 1 Description of the tuberculosis outbreak (four cases of tuberculosis and 11 individuals with latent tuberculosis infection) observed in Lecce Province, Puglia (Italy) in 2014. CXR: chest radiography; TST: tuberculin skin test; IGRA: interferon- $\gamma$ release assay; CT: computed tomography; MIRU: mycobacterial interspersed repetitive units; AFB: acid-fast bacillus.

Out of the 35 additional immigrants tested, five were TST positive but chest radiography negative. All of the nine immigrant association staff were TST negative.

All the 22 nursery school staff plus the three kitchen staff were TST negative. Out of the 90 children attending the nursery school, a female child (case C, classmate of case A) was IGRA positive on May 28, 2014. Having an apical lesion at the chest radiography (small nodule in the lower lobe of the right lung), although negative for AFB in her gastric aspirate, she started standard anti-TB treatment as per WHO guidelines [8, 9].

In the contact tracing of case C, while 21 external contacts were TST negative, three out of 17 family members yielded a positive TST. While a 5-year-old female (cousin of case C) was IGRA positive and chest radiography negative, case C's mother (34 years of age, case D) had a chest radiography compatible with TB and was AFB positive.

Case D was hospitalised in the infectious diseases department of Santa Caterina Novella Hospital in Galatina (Lecce Province). The diagnostic delay (6 months) was caused by case D's delay in reporting her signs and symptoms (cough, fever and loss of weight) to a physician until 4 months after delivery, when she underwent the first chest radiography and magnetic resonance $(7-\mathrm{cm}$ cavity in the upper right lung with several nodular lesions in the left one). She was AFB positive and was prescribed standard anti-TB regimen.

Samples were sent for culture and drug susceptibility testing (DST) to the Lecce hospital laboratory using the existing intra-province sample transportation mechanism. The results were available after 30 and 58 days of treatment, respectively. The DST showed no resistance. The Lecce laboratory performed both 
tests on solid culture (results provided within 30-60 days) $\left({ }^{\mathrm{d} 2}\right)$. No molecular rapid tests, like line probe assay (LPA) or Xpert MTB/Rif (Cepheid, Sunnyvale, CA, USA) are available in any of the laboratories of Lecce Province $\left({ }^{\mathrm{d} 1}\right)$. Her MIRU-VNTR24 (also available from "S. Raffaele" Institute, Milan, in November 2014) showed identical results to that of case A.

The younger daughter of case D, screened as all close contacts of case C, having a negative chest radiography, initially underwent treatment for latent TB infection (LTBI) with isoniazid $100 \mathrm{mg}$ per day. After 43 days of LTBI treatment, culture on solid medium was positive for M. tuberculosis (case E). She was admitted and the CT scan showed a "lesion in the upper portion of the lobe of the left lung". She was then prescribed anti-TB treatment: HRZE for 8 weeks followed by HRZ in the continuation phase (because of concerns about possible development of resistance to $\mathrm{H}$ after over a month of $\mathrm{H}$ monotherapy until culture results became available). $\mathrm{Z}$ was used instead of $\mathrm{E}$ in the continuation phase, taking into consideration case E's young age (inability to report visual impairment) and the limited extension of the lung lesion (detected only by CT scan).

This outbreak included four TB cases (all native Italians without specific risk factors, with case D probably being the index case) and 11 LTBIs plus one unrelated case in a foreign-born individual (eight TST-positive cases related to case A and three IGRA-positive cases related to case C). All LTBI individuals were treated. Informed consent was obtained.

Two deviations from established recommendations were noted [8,9], being related to the use of suboptimal diagnostic tools for TB detection $\left({ }^{\mathrm{d} 2}\right.$ ) (liquid culture being the gold standard for TB diagnosis) and the lack of rapid diagnostics of drug-resistant TB $\left({ }^{\mathrm{d} l}\right)$. The use of amikacin was not interpreted as a deviation, as it was intended to treat pneumonia and not to exclude TB.

The Puglia region, with $>4$ million inhabitants, is administratively divided into six provinces and correspondent health directorates, each one with at least one main secondary care hospital with a laboratory performing direct smear examination and culture (using either solid or liquid media to detect M. tuberculosis). The Lecce laboratory is neither equipped with liquid culture nor with rapid diagnostics for drug-resistant TB (Xpert MTB/RIF or LPA). These tools are available in other laboratories, including Bari University hospital located in the regional capital. No mechanism is in place to transport the samples outside each province and no "reference" regional laboratory has been officially identified.

The lack of access to modern diagnostic tools for TB diagnosis resulted in delayed information on culture and sensitivity to anti-TB medications. The treatment of case E as LTBI instead of as an active case has resulted in potential acquisition of resistance and worse prognosis. No genotypic study to study the outbreak was possible locally, with MIRU performed later in a laboratory in the north of the country and correspondent results being available almost 1 year later. The local public health services investigating on this outbreak had to identify, firstly, where to perform such investigations, and then, how to send the samples and pay for the testing.

This report highlights how TB keeps circulating among native Italians, the lack of access to internationally recommended diagnostic TB services being a potential contributing factor. Political commitment and better health service coordination, advocated in the new WHO End TB Strategy $[1,10]$, are imperative if we really want to prevent the emergence of drug resistance $[11,12]$, and target TB Elimination in Italy and in Western Europe [13, 14].

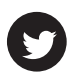

@ERSpublications

Managing tuberculosis outbreaks in Southern Italy is difficult, hampering elimination efforts http://ow.ly/JE7dy

Laura Gillini ${ }^{1}$, Rosella Centis ${ }^{2}$, Lia D’Ambrosio ${ }^{2}$, Alberto Fedele ${ }^{3}$, Valerio Aprile ${ }^{3}$, Giancarlo Pasanisi ${ }^{4}$, Leonardo Donateo ${ }^{5}$, Danila Costa ${ }^{6}$, Giovanni Battista Migliori ${ }^{2}$ and Paolo Tundo ${ }^{7}$

${ }^{1}$ World Health Organization, Division of Pacific Technical Support, Suva, Fiji. ${ }^{2}$ World Health Organization Collaborating Centre for Tuberculosis and Lung Diseases, Fondazione S. Maugeri, Care and Research Institute, Tradate, Italy. ${ }^{3}$ Prevention Dept, Hygiene and Public Health Service, Local Health Agency, Lecce, Italy. ${ }^{4}$ Laboratory Medicine Dept, "Card. G. Panico" Hospital, Tricase, Italy. ${ }^{5}$ Executive Pneumology and Tuberculosis Control Services, Lecce Health Directorate, Lecce, Italy. ${ }^{6}$ Microbiology and Virology Unit, Mycobacteria laboratory, University Hospital, Policlinico, Bari, Italy. ${ }^{7}$ Infectious Diseases Dept, "S. Caterina Novella” Hospital, Galatina, Italy.

Correspondence: Giovanni Battista Migliori, World Health Organization Collaborating Centre for Tuberculosis and Lung Diseases, Fondazione S. Maugeri, Care and Research Institute, via Roncaccio 16, 21049, Tradate (VA), Italy.

E-mail: giovannibattista.migliori@fsm.it

Received: Jan 232015 | Accepted after revision: Feb 162015 | First published online: May 292015

Conflict of interest: None declared. 


\section{References}

1 SotgiuG, Mauch V, Migliori GB, et al. Evidence-based, agreed-upon health priorities to remedy the tuberculosis patient's economic disaster. Eur Respir J 2014; 43: 1563-1566.

2 Voniatis C, Migliori GB, Voniatis M, et al. Tuberculosis elimination: dream or reality? The case of Cyprus. Eur Respir J 2014; 44: 543-546.

3 Diel R, Loddenkemper R, Zellweger JP, et al. Old ideas to innovate tuberculosis control: preventive treatment to achieve elimination. Eur Respir J 2013; 42: 785-801.

4 D'Ambrosio L, Dara M, Tadolini M, et al. TB Elimination: theory and practice in Europe. Eur Respir J 2014; 43: $1410-1420$.

5 Lönnroth K, Migliori GB, Abubakar I, et al. Towards tuberculosis elimination: an action framework for low-incidence countries. Eur Respir J 2015; 45: 928-952.

6 Esposito S, D'Ambrosio L, Tadolini M, et al. European Respiratory Society/World Health Organization Tuberculosis Consilium assistance with extensively drug-resistant tuberculosis management in a child: case study of compassionate delamanid use. Eur Respir J 2014; 44: 811-815.

7 Codecasa LR, Ciconali G, Mazzola E, et al. Managing an XDR-TB outbreak: the public health face of the medal. Eur Respir J 2015; 45: 292-294.

8 van der Werf MJ, Sandgren A, D'Ambrosio L, et al. The European Union standards for tuberculosis care: do they need an update? Eur Respir J 2014; 43: 933-942.

9 Falzon D, Jaramillo E, Schünemann HJ, et al. WHO guidelines for the programmatic management of drug-resistant tuberculosis: 2011 update. Eur Respir J 2011; 38: 516-528.

10 World Health Organization. Global Tuberculosis Report 2014. Document WHO/HTM/TB/2014.08. Geneva, World Health Organization, 2014.

11 Migliori GB, Sotgiu G, Gandhi NR, et al. Drug resistance beyond extensively drug-resistant tuberculosis: individual patient data meta-analysis. Eur Respir J 2013; 42: 169-179.

12 Falzon D, Gandhi N, Migliori GB, et al. Resistance to fluoroquinolones and second-line injectable drugs: impact on multidrug-resistant TB outcomes. Eur Respir J 2013; 42: 156-168.

13 Smit PW, Vasankari T, Aaltonen H, et al. Enhanced tuberculosis outbreak investigation using whole genome sequencing and IGRA. Eur Respir J 2015; 45: 276-279.

14 Abubakar I, Matthews T, Harmer D, et al. Assessing an outbreak of tuberculosis in an English college population. Eur Respir J 2011; 38: 976-978.

\section{Discrepant elevation of sIL-2R levels in sarcoidosis patients with renal insufficiency}

To the Editor:

Sarcoidosis is a systemic, granulomatous disease that can manifest in multiple organs [1]. Several biomarkers are used to assess disease activity and monitor response to therapy, including soluble interleukin-2 receptor (sIL-2R) and angiotensin-converting enzyme (ACE) [2]. sIL-2R was previously shown to correlate with the amount of $\mathrm{CD}^{+}$T-lymphocytes in bronchoalveolar lavage fluid [3]. Furthermore, it was shown that sIL-2R $>4000 \mathrm{pg} \cdot \mathrm{mL}^{-1}$ was a significant predictor of relapse after discontinuation of infliximab therapy and that sIL-2R is a suitable prognostic marker for disease progression $[4,5]$. We observed, however, that in a small number of patients with co-existing renal insufficiency, sIL-2R can be disproportionately high without marked signs of disease activity based on ${ }^{18}$ F-fluorodeoxyglucose positron emission tomography (FDG-PET), ACE and the clinical presentation. The aim of this pilot study is therefore to evaluate the influence of renal function on sIL-2R levels to determine if and how the marker can be used in sarcoidosis patients with renal insufficiency. In order to further illustrate the discrepancy, we first describe two cases.

The first case concerns a 56-year-old woman with pulmonary sarcoidosis, and a history of nephrotic syndrome and membranous glomerulonephritis resulting in moderate renal insufficiency (estimated glomerular filtration rate (eGFR) $50 \mathrm{~mL} \cdot \mathrm{min}^{-1}$ per $1.73 \mathrm{~m}^{2}$ ). ACE levels were repeatedly normal over a period of $>2$ years and no signs of sarcoidosis disease activity were present on the latest FDG-PET scan. However, sIL-2R levels remained highly elevated ( $>9000 \mathrm{pg} \cdot \mathrm{mL}^{-1}$; normal reference value $<3000 \mathrm{pg} \cdot \mathrm{mL}^{-1}$ ) despite treatment with infliximab.

The second case involves a 50-year-old man with sarcoidosis and a complicated history of stage $\mathrm{V}$ diabetic nephropathy, heart failure and various infections. The patient was treated with peritoneal dialysis. ACE was normal but sIL-2R levels were repeatedly extraordinarily high $\left(>50000 \mathrm{pg} \cdot \mathrm{mL}^{-1}\right)$ despite immunosuppressive 